\title{
Imaging Material Response During Laser Modification of Fused Silica
}

\author{
Rajesh N. Raman, Raluca A. Negres, and Stavros G. Demos \\ Lawrence Livermore National Laboratory, 7000 East Ave, Livermore, CA 94551
}

High average power lasers are essential for applications such as microlithography, micromachining, laser welding, and laser-driven inertial confinement fusion (ICF). Laser-induced damage in optical materials remains a critical problem in these applications; optical energy densities, although not approaching the breakdown threshold of the components, can cause catastrophic failure of the optical components via laser-induced damage and subsequent damage growth. It is believed that laser-induced damage initiates at pre-existing or laser-induced defective regions in the material (precursors) that act as centers of absorption of the incident light energy in an otherwise normally transparent (defect-free) material [1]. The resulting morphology of the damage site results from the material's response to localized laser energy deposition. However, the timeline of events leading up to this end result has not been documented with sufficient resolution. We hypothesized that characterizing the early material modifications will improve our current understanding of the nature of the precursors and methods of energy deposition. In this work, we demonstrate an experimental system that allows for imaging the material response during laser-induced damage with adequate spatial and temporal resolution. This experimental system employs a suite of configurations, each optimized to capture specific aspects of the timeline of events during damage of the exit surface of fused silica by nanosecond-scale laser pulses.

Experiments were executed on a circular fused silica sample $5 \mathrm{~cm}$ in diameter and $1 \mathrm{~cm}$ in thickness. Damage sites were created by a (pump) laser operating at $355 \mathrm{~nm}(3 \omega)$ and producing 7-ns FWHM pulses, focused about $2 \mathrm{~cm}$ behind the exit-surface of the sample for a resulting beam diameter of $\sim 1 \mathrm{~mm}$ at the surface. Pristine surface sites were first exposed to a pump pulse having an average fluence of $\sim 30 \mathrm{~J} / \mathrm{cm} 2$, leading to the formation of single or multiple pit craters on the order of $10-50 \mu \mathrm{m}$ in diameter on average.

To capture early material response to laser energy deposition during exposure to the pump pulse, a high temporal resolution microscopy system was developed. Shadowgraphic images were captured by means of illumination by a (probe) laser operating at $532 \mathrm{~nm}(2 \omega)$ and producing pulses $150 \mathrm{ps}$ in length FWHM (Figs. 1a-b). During exposure to pulses of this length, material moving close to the speed of sound in fused silica can be resolved with the same $(\sim 1 \mu \mathrm{m})$ resolution achieved in the static imaging case. Two probe pulses, delayed by nanoseconds with respect to each other, were generated in order to probe two time points of the same event; this was achieved by splitting the probe laser output into two paths with a beam-splitter and directing the reflected beam (probe 2) to an adjustable delay line. The pulses were differentiable by means of a polarization filtering method. Time $\tau$ above each image represents the peak-to-peak delay of the probe pulse relative to the pump pulse. It was observed that during the pump pulse, small absorptive sites appear before the peak of the pump pulse is reached ( $\tau=0)$ (Fig. 1a). However, the dimensions of the resulting damage crater long after the pump pulse presented in Fig. 1b shows that these sites grow to their final size at a later time.

Sensitivity to small mass motion at the surface was achieved by imaging the event in a backscattering configuration: the probe illumination was directed onto the exit-surface at $\sim 60^{\circ}$ angle of incidence, and light scattered from the material was imaged directly onto a camera (dark-field) viewing the sample from the side. Towards the end of the pump pulse, a swelling of the surface appears, leading to its expansion on the order of few tens of micrometers. Figure 1c shows material swelling (towards the left-hand side) at $\sim 25 \mathrm{~ns}$ delay.

Ejecta kinetics were determined by probing the material with two probe pulses at two different time points during each event to allow for the tracking of specific particles along their trajectories After $\sim 50 \mathrm{~ns}$ delay, material ejection is observed as a jet consisting at first of small ( $\varnothing \sim 1-4 \mu \mathrm{m}$-diameter) particles with speeds up to $v \sim 3 \mathrm{~km} / \mathrm{s}$. At later delays, average particle size increases and average speed decreases. For example, at $\sim 250 \mathrm{~ns}$ delay, particles with diameters $\sim 1-15 \mu \mathrm{m}$ and speeds up to $\sim 2 \mathrm{~km} / \mathrm{s}$ are observed (Fig. 2). Material clusters advance in fronts of similar speed with later fronts characterized by slower speeds. The ejection process continues until about $\tau=10 \mu$ s. This work was performed 
under the auspices of the U.S. Department of Energy by Lawrence Livermore National Laboratory under Contract DEAC52-07NA27344.

[1] L. Skuja, J. Non-Cryst. Solids 239 (1998) 16.

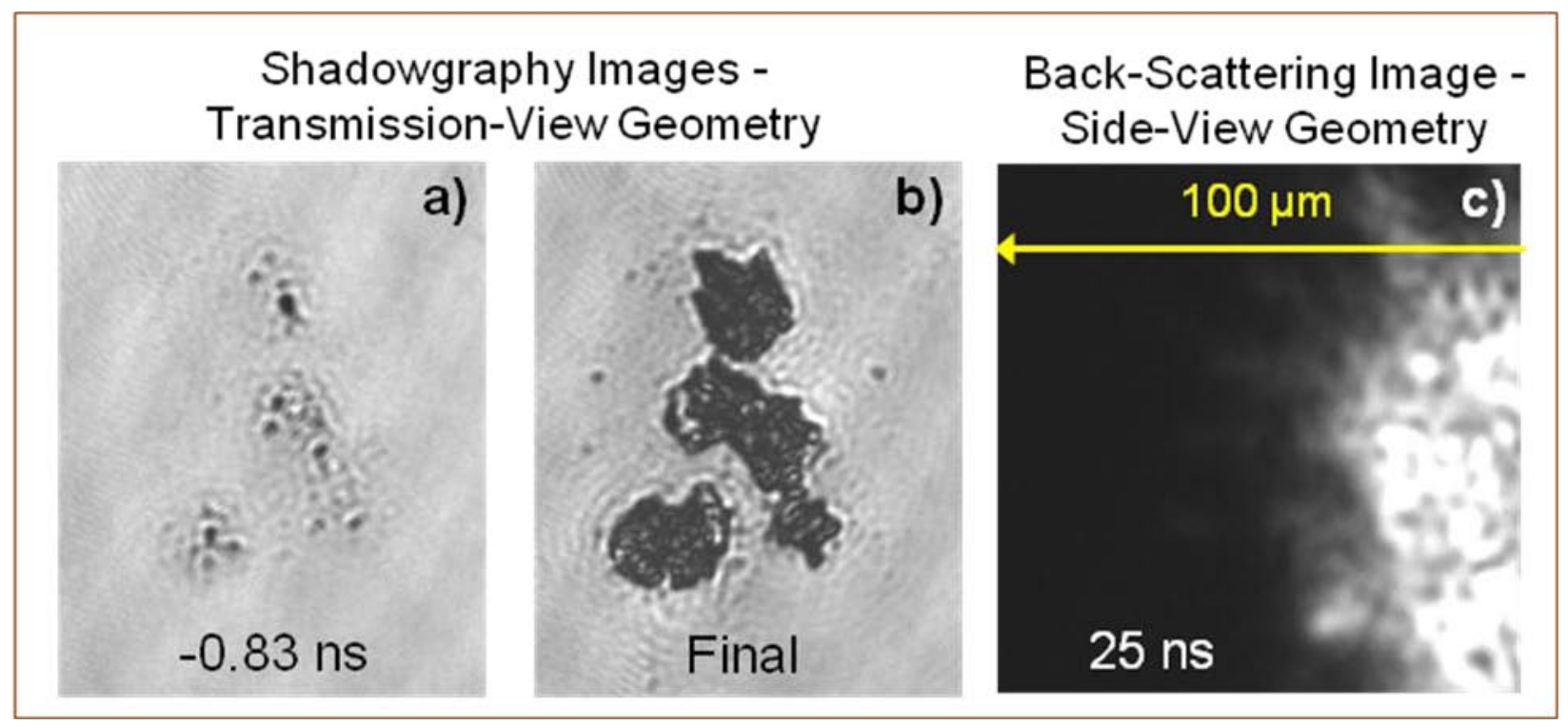

FIG. 1. (a-b) Transmission shadowgraphy images of a damage event a) during and b) after exposure to the pump pulse. c) Back-scattering image of the side-view of the surface (located on the right-hand side) at 25 ns delay.

\section{Shadowgraphy Image - Side-View Geometry}

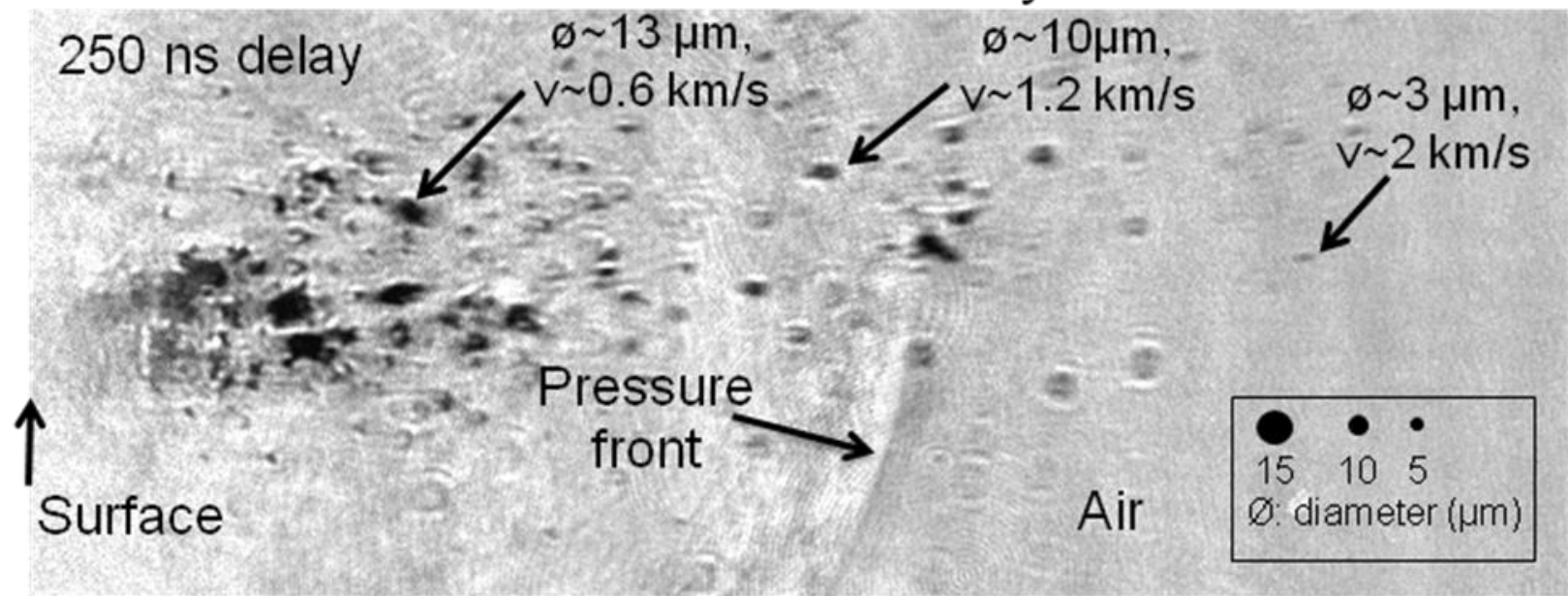

FIG. 2. Shadowgraphy image of the sample as viewed from the side at $250 \mathrm{~ns}$ delay. The diameter and speed of several ejected particles (traveling toward the right) are indicated, along with the location of a pressure front. 